\title{
CARPIUC benchmark overview: crack advance, reorientation, propagation and initiation under complex loadings
}

\author{
Andreea Carpiuc ${ }^{1}$, Martin Poncelet ${ }^{2^{*}}\left(\mathbb{D}\right.$, Julien Réthoré ${ }^{3}$ and Stéphane Roux ${ }^{2}$
}

${ }^{*}$ Correspondence:
martin.poncelet@ens-paris-
saclay.fr
${ }^{2}$ LMT (ENS
Paris-Saclay/CNRS/Univ.
Paris-Saclay), 61 Avenue du
Président Wilson, 94235 Paris,
Cachan, France
Full list of author information is
available at the end of the article

\begin{abstract}
A series of experiments has been performed to build a benchmark for the numerical modeling of mixed mode crack propagation in concrete. Two tests are selected so that the propagation is almost always stable although the material is quasi-fragile. Moreover the variation of mode mixity enables the study of the reorientation of the crack, as well as more complex phenomena such as branching or link-up. To control stability and mode mixity, the loading is imposed with a 6 -axis testing machine while the crack propagation is assessed by full-field measurement. In this paper, a description of the experimental setup is provided as well as numerical simulation results illustrating the sensitivity to boundary conditions and geometry. Links to data needed to perform numerical simulations of these benchmark experiments are given, together with references to publications giving full descriptions of the protocol and experiment results.
\end{abstract}

Keywords: Crack, Concrete, Mixed-mode, Full-field measurement, Numerical model validation, Boundary conditions

\section{Introduction}

Numerically predicting mixed-mode crack propagation for quasi-brittle materials is a complex task. First, from a theoretical point of view, the modelling of the behaviour of the crack tip in itself and of the contact/friction of the crack surface is known to be challenging. Second, from a numerical point of view, taking into account an evolving (quasi)singularity very close to instabilities requires sophisticated tools. The validation of computation results through a quantitative comparison with experimental results is thus a mandatory step prior to using such numerical models in an industrial context.

One is then confronted with the lack or limitations of the existing test results. The most widespread tests use a single actuator and a complex specimen shape: $\mathrm{L}$-shaped geometries [1], asymmetric four-point bending (pioneer work of Iosipescu [2]), out-of-plane bending [3]. The ratio between the stress intensity factors (SIFs) cannot be controlled using a single actuator, and it is entirely depending on the initial geometry and boundary conditions (BC) of the specimen. This limitation can be circumvented by using two actuators, although such a possibility is much more demanding experimentally, and hence much more rare: two control point bending [4] or double-notched specimens under independent tension

(c) The Author(s) 2018. This article is distributed under the terms of the Creative Commons Attribution 4.0 International License (http://creativecommons.org/licenses/by/4.0/), which permits unrestricted use, distribution, and reproduction in any medium, provided you give appropriate credit to the original author(s) and the source, provide a link to the Creative Commons license, and indicate if changes were made. 
and shear [5]. These are the only ones which allow controlled SIF histories, including non-proportional ones.

The case of independent tension and shear, despite several drawbacks (no validation of pure in-plane loading, lack of some material parameters, no full-field measurements...), has been extensively used for numerical validation (see e.g., [6-18]). Thus, designing modern tests, inspired by this initial tension-shear setup, contributes to the development of more reliable simulations of mixed-mode crack propagation in brittle materials.

In the present experiments, the loading is applied by a 6-axis testing machine [19], so that in addition to the global tension and shear of the specimen, a gradient of tension can be applied. Moreover, loadings that may lead to out-of-plane motion can be controlled. The displacement of the specimen is at the same time measured with a full-field technique (here digital image correlation (DIC) [20]), capturing the crack path and tip along the test, rather than only at the final stage (post-mortem). The combination of the control of the tension gradient and of measurement of the crack tip position offers the opportunity to perform complex SIF histories-and hence complex crack paths-by modifying the loading during the test.

The question of the way the experimental results have to be implemented into a numerical code to be relevant must also be raised. Consequently several simulations using a damage model are performed with different $\mathrm{BC}$ to study their influence. The notches description is studied as well as the resolution of the imposed displacement field on the boundary.

The purpose of this paper is to provide an overview of the CARPIUC benchmark. For the sake of brevity, more technical and detailed presentations will be pointed at using published or to-be-published references, as well as the links to data needed to perform numerical simulations. Only a brief summary will be provided with the ambition of giving the general guidelines of this approach which gathers experimental tests and numerical simulations of them. The first part of this paper presents the experimental protocol, the second the results of the two tests, and the third part is a discussion on the relative importance of the $\mathrm{BC}$ type, the notch geometry and the crack initiation in the case of one specific damage numerical model.

\section{Experimental protocol}

\section{Specimens}

The specimens are made of mortar, whose composition consists of $0 / 4$ sand, Portland cement and water $(w / c=0.42)$ with plasticizer. Their geometry is similar to the one used by [5], apart from the presence of a single notch in one test case (Fig. 1). The top and bottom ends of the specimen are glued $24 \mathrm{~h}$ before the test to monolithic steel $\mathrm{U}$-shaped parts that are fixed to the testing machine.

The mechanical properties are obtained following the standard 196-1 NFEN [21]: Young modulus, tensile strength and compressive strength respectively equal to $17.25 \pm 0.74 \mathrm{GPa}$, $3.8 \pm 0.4$ and $80 \mathrm{MPa}$. The fracture energy, $G_{f}$, as obtained with $70 \times 70 \times 280 \mathrm{~mm}^{3}$ specimens following the [22] standard guidelines, is equal to $114.6 \pm 18.8 \mathrm{~J} / \mathrm{m}^{2}$. More details concerning these characterization tests may be found in [23]. 


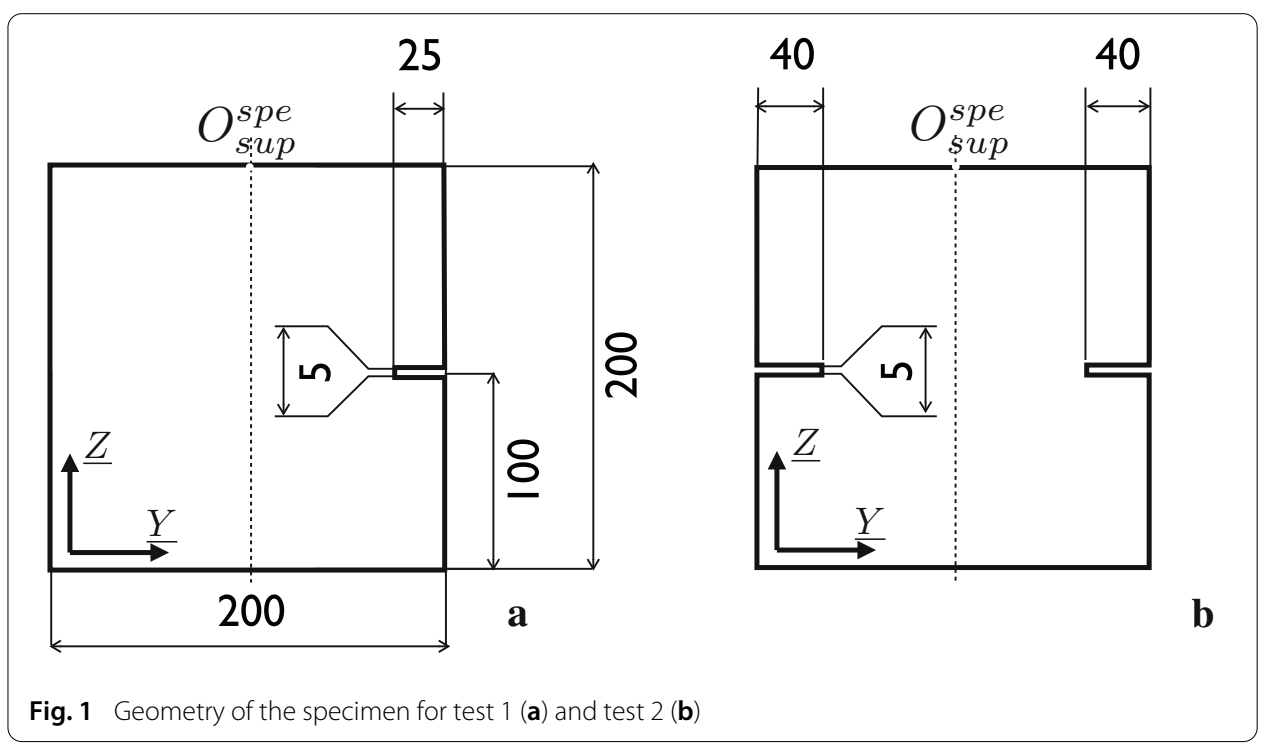

Fig. 1 Geometry of the specimen for test 1 (a) and test 2 (b)

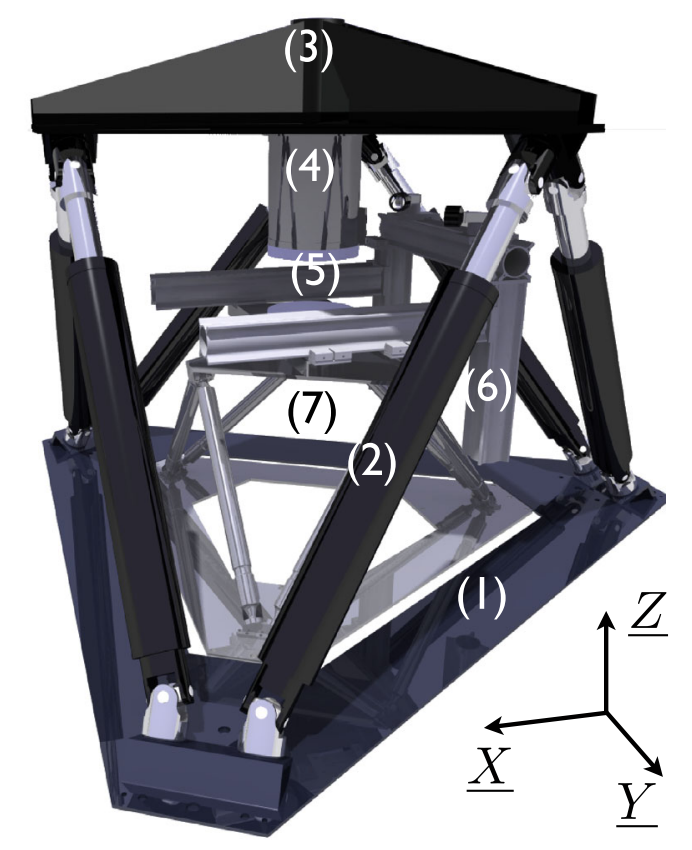

Fig. 2 The 6-axis testing machine: (1) base, (2) actuators, (3) moving platform, (4) upper end-effector, (5) room for specimen, (6) optical setup in close configuration with two cameras, (7) passive hexapod

\section{Testing machine}

A 6-axis electromechanical testing machine $[19,24]$, called "Hexapod", is used for the test (see Fig. 2). Since it is a parallel architecture machine, the six components (3 forces and 3 torques) of the load capacity are limited to a domain that is position dependent. Values are thus given here, in the case where only one component is non-zero: $120 \mathrm{kN}$ and $70 \mathrm{kNm}$ along $\underline{Z}, 50 \mathrm{kN}$ and $45 \mathrm{kNm}$ in the $\underline{X}-\underline{Y}$ plane. The accessible displacement and rotation domain has a complicated shape, but is roughly contained in a box of amplitude 
$\pm 250 \mathrm{~mm}$ for displacements and $\pm 22^{\circ}$ for rotations. The 6 -axis loadcell, in the form of a small hexapod (label (7) in Fig. 2) has measurement uncertainties equal to $80 \mathrm{~N}$ and $20 \mathrm{Nm}$.

To compensate the clearances and compliances of the different parts of the machine, and for accurate displacement measurement under load, a dedicated digital image correlation (DIC) technique is used: three cameras, fixed to the bottom part of the specimen, are aimed at three targets fixed to the upper part. Synchronized image acquisition and analysis through DIC provide displacements with an error as low as $1 \mu \mathrm{m}$ over a $[-100 ; 100] \mu \mathrm{m}$ range in any direction as discussed in [19].

\section{Instrumentation}

Different measurement techniques were used (LVDT, 2D DIC, stereo-DIC), but the results shown here only rely on the 2D full field kinematic measurement of each $200 \times 200 \mathrm{~mm}^{2}$ face of the specimens. The other techniques were mainly used to check that the tests were-as expected-in-plane ones.

The presented 2D DIC results are obtained with a regularized global DIC approach [26] and one camera $(2048 \times 2048$ pix., 12 bits, pixel physical size $\approx 130 \mu \mathrm{m})$ for each side. Images are synchronized and captured every $5 \mathrm{~s}$. The DIC mesh element size is 15 pix. and the regularization length is equal to 250 pix. The standard displacement uncertainty is about 1.3 (resp. 2.4) $\mu \mathrm{m}$ along $\underline{Z}$ (resp. $\underline{Y}$ ) direction. A more detailed description of the protocol may be found in [23].

\section{Loadings}

In order to create complex crack paths a combination of proportional and nonproportional elementary displacements are applied to the upper face of the specimen while the bottom face is fixed as shown in Fig. 3:

- A global tension (i.e., vertical translation).

- A global shear (i.e., horizontal translation).

- A gradient of vertical displacement (i.e., in-plane rotation).

This setup was used to replicate some of the reference Nooru-Mohamed tests [27], (namely no 6a and no 5 loadings from this reference were repeated respectively four and three times) with full-field displacement measurements. These tests are not proposed for the benchmark, but the interested readers may find further information in [28]. Other more complex tests including rotations, in-plane crack initiation, propagation, reorientation, branching or linkup scenario and even coupling to mode III were also performed, and are described extensively in [29].

Among these experiments [29], two tests are selected and proposed for the CARPIUC benchmark. A displacement rate of approximatively $0.01 \mu \mathrm{m} / \mathrm{s}$ is applied, corresponding to quasi-static loading conditions. Rotation is used to make crack propagation more stable for a much longer time, enabling a live control of the crack orientation. The crack propagation appears to be stable during almost the entire test (several hours), only a brief period of unstable crack propagation is observed at the end of the test. The latter is a dynamic event that results in the complete failure of the specimen. Crack evolution is controlled during the test, using the previously cited DIC technique [26] and images captured by the 


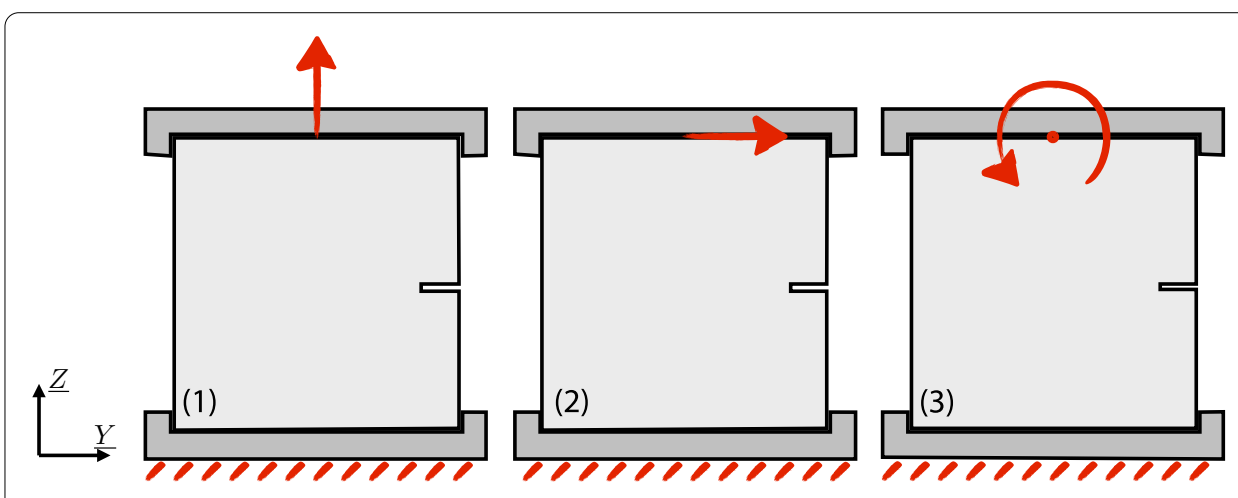

Fig. 3 The three elementary loading paths: 'tension' (1), 'shear' (2) and 'rotation' (3)

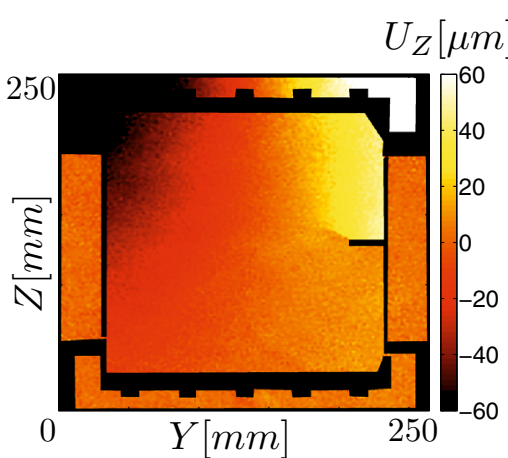

a

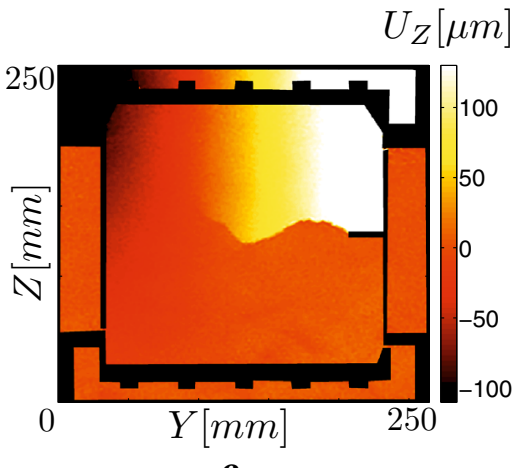

c

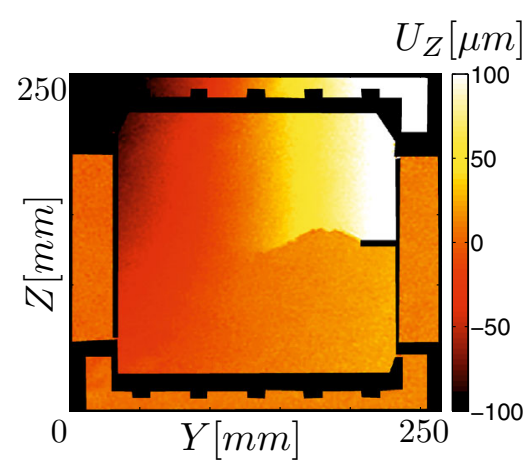

b

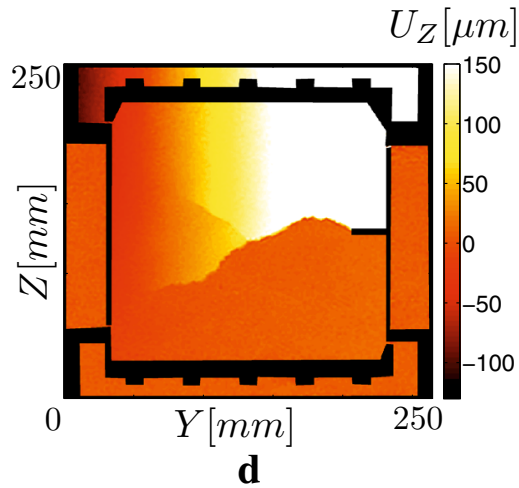

Fig. 4 Vertical displacement fields showing the crack advancement for test no 1 after each propagation step. Loading plates glued to the upper and lower ends of the specimen are visible

cameras pointing to the faces. To follow a desired crack propagation scenario, the loading is monitored to reorient the crack (possibly leading to branching) in order to follow the crack tip predefined positions along the crack path.

\section{Experimental results}

\section{Test no 1}

The first test case of the benchmark consists of a single crack initiation, followed by several reorientations and finally crack branching, resulting in a jagged crack path. The sample 


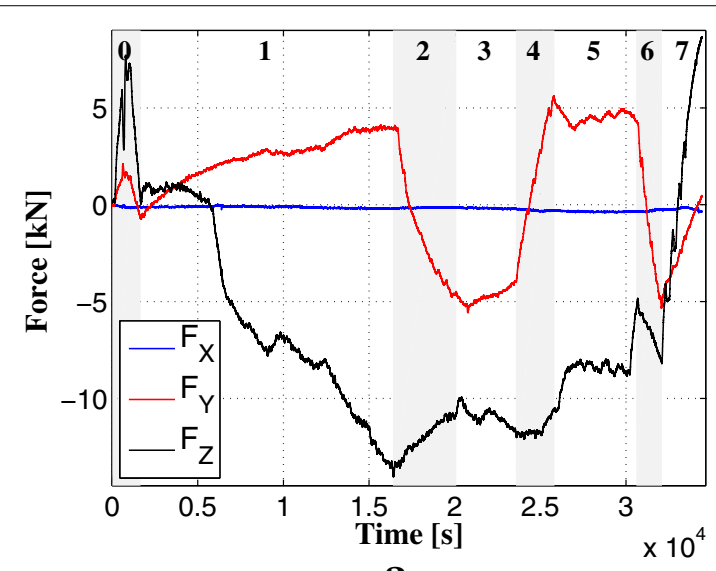

a

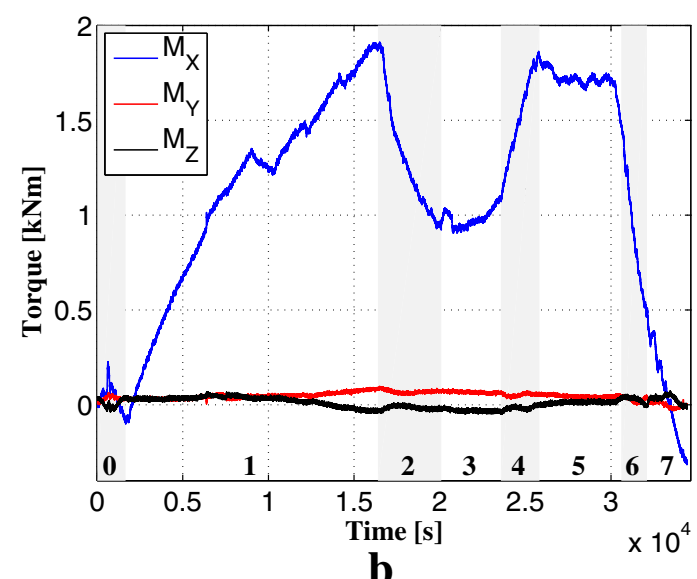

Fig. 5 Forces (a) and torques (b) measured during test no 1 and expressed at point $O_{\text {sup }}^{\text {spe }}$

geometry is chosen with one single notch (Fig. 1a). The loading consists of a series of tension, rotation and shear steps (Fig. 3) to initiate the crack, and to (attempt to) drive it along a predefined path. At each loading step, a branching may occur from a corrugation of the current crack path rather than desired crack tip advance in a specific direction, and the risk of branching increases with the crack length. Thus the obtained crack path has roughly the sought zigzag geometry eventually interrupted by a branching and brutal propagation until complete failure. The final crack at the end of each propagation step is given in Fig. 4. The forces and torques measured during the test are shown in Fig. 5.

During the test, crack closure is observed. However, it may be considered as negligible because simulations performed with a non-local damage model [31] presented thereafter with and without stiffness recovery give good results in terms of crack path prediction in both cases. However, the force-displacement curves obtained with the model considering the stiffness recovery are closer to the experimental data than the ones obtained without any crack closure formulation (for more details, see [23]).

\section{Test no 2}

The second test presents a double initiation: two cracks (one from each notch) propagate toward each other, until coalescence (linkup). It also addresses crack closure and friction 


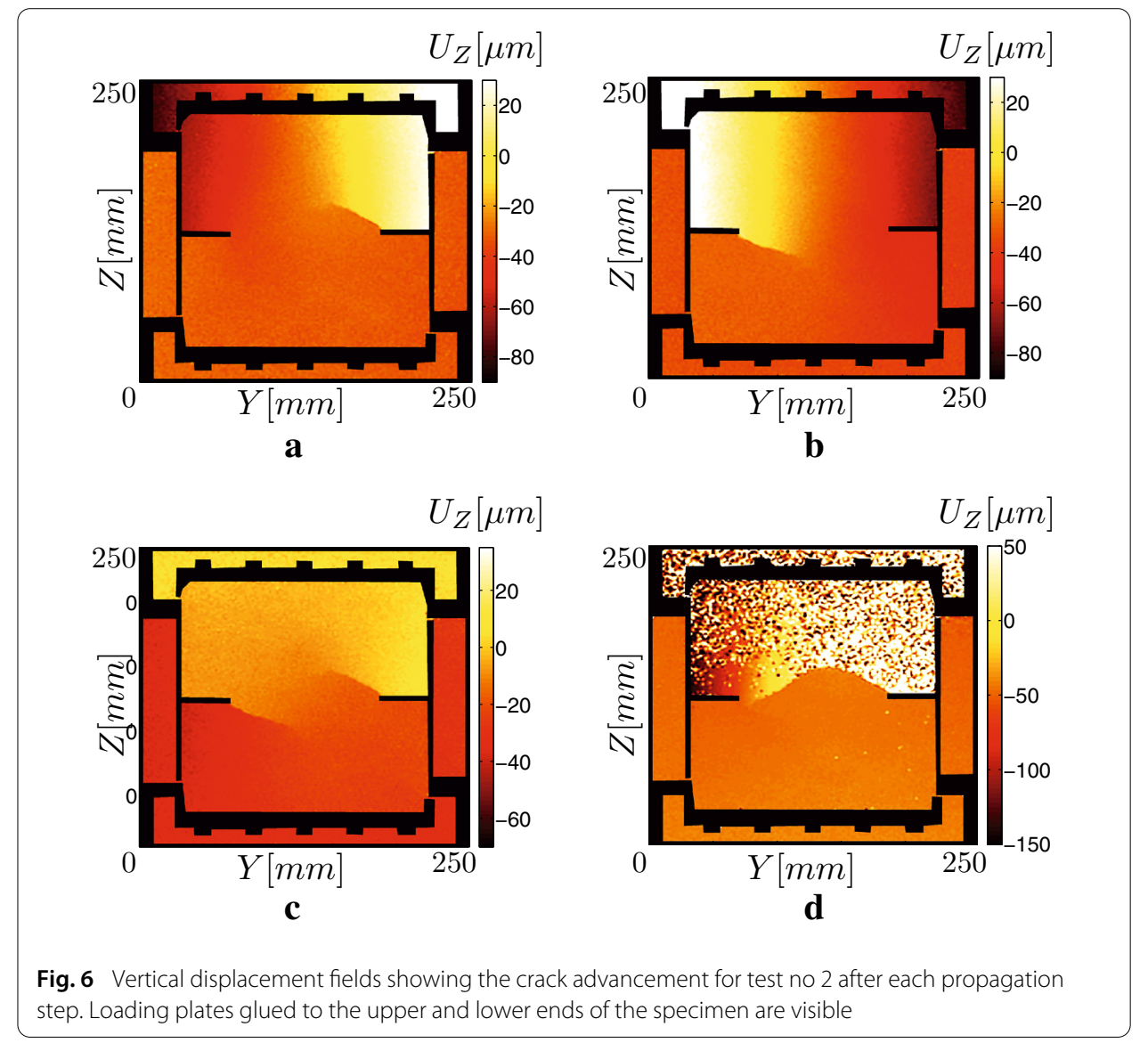

phenomena. The chosen geometry is the one with two notches (Fig. 1b). The test can be divided into four loading steps composed of combinations of elementary loading paths like tension, shear and in-plane rotation (Fig. 3). More precisely the test starts by applying positive in-plane rotation combined with a positive shear force to initiate and propagate a crack upward. Afterward, the system is unloaded and reloaded by coupling a negative in-plane rotation with a positive shear force leading to the closure of the crack and the initiation of a second crack on the opposite notch. After a second unloading, the final step is a proportional tensile-negative shear loading that reorients both cracks and links them up. The evolution of the crack after each propagation step and the evolution of the measured forces and torques are respectively given in Figs. 6 and 7.

Even though during the propagation of the second crack, the first one is submitted to a small compressive strain, when comparing the crack paths predicted by a non-local damage model [31] (presented in Sect. Methodology) considering crack closure with those predicted without the crack closure formulation, almost no difference is noted. The forces and torques are also close for both formulations (for more details, see [25]). Therefore, accounting for unilateral contact is not a pre-requisite for modelling this test.

\section{Numerical implementations of the boundary conditions}

Boundary conditions $(\mathrm{BC})$ are very important when performing the numerical simulations of complex experimental tests. The majority of the cases reported in literature are 


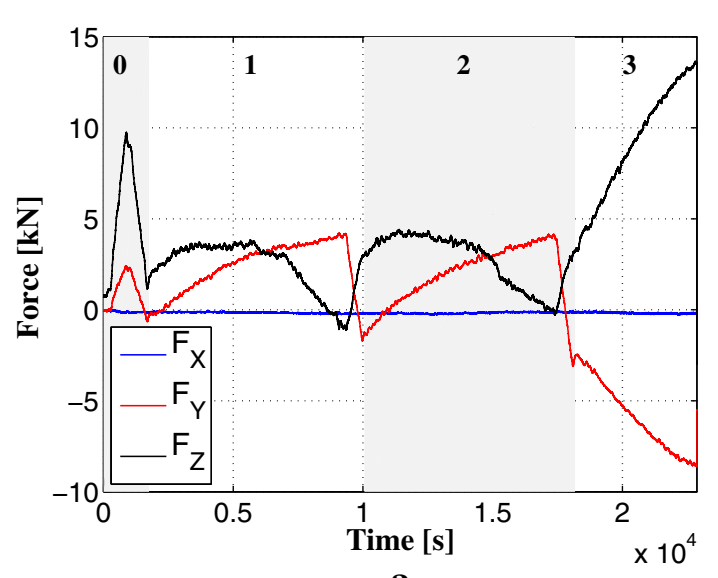

a

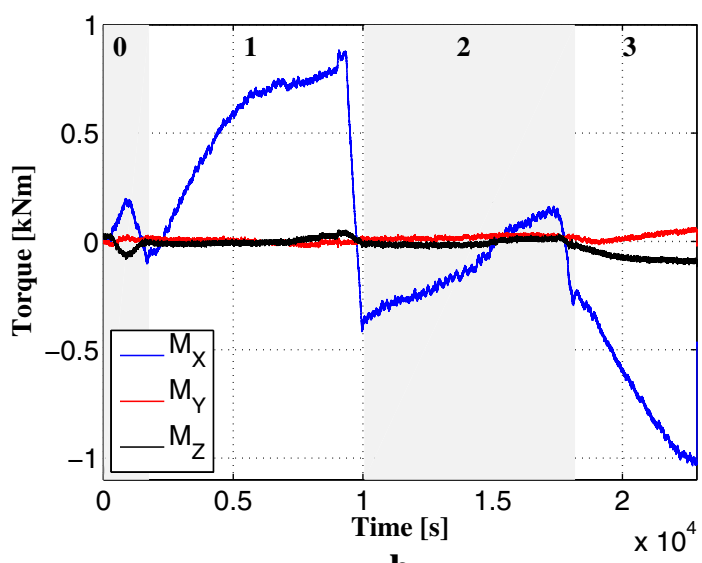

b

Fig. 7 Forces (a) and torques (b) measured during test no 2 and expressed at point $O_{\text {sup }}^{\text {spe }}$

instrumented using point measurements and the models are tested using simple scalar data $[8,13,16,17]$. For the proposed tests displacement fields are measured on the specimen and on the loading plates. Therefore, the choice of the $\mathrm{BC}$ is both very important and not straightforward, as thoroughly shown in $[25,30]$. It is here proposed to report some observations from these early studies in order to guide people who would be interested in participating to the benchmark. First, a brief description of the model used for the simulations is given. Then, four main points are discussed: the choice of $\mathrm{BC}$ corresponding to the displacement of the loading plates or measured directly on the sample, the choice of the thickness of a purely elastic layer at the sample boundary, the choice of more or less detailed BC (full-field vs. scalar) and the choice of the notch geometry.

\section{Methodology}

To figure out how experimental data have to be used in numerical simulations, a non-local damage model $[31,32]$ has been used. This model describes the degradation behavior of quasi-brittle materials, like concrete, by using a scalar damage field $a(0 \leq a \leq 1)$. Moreover, one advantage of using a damage model is that the crack initiation location is predicted by the model without the need of an initial artificial crack, and the trajectory is 
not predefined (as in the case of some cohesive zone models). The scalar damage model is thus a good compromise for the following study.

The damage evolution is governed by an energy function taking into account the gradient of the damage field, so that the usual numerical issues regarding mesh dependence due to damage localization are inherently solved. The energy function takes also into account the elastic domain, which is described by a criterion proposed by [33]. It enables to accurately describe the assymmetric behavior in tension and compression, and also in shear [31]. The stiffness recovery is also accounted for by the model.

\section{$B C$ from the loading plates vs. from the samples}

Two types of $\mathrm{BC}$ are studied (Fig. 8). The first ones are captured from the loading plates and applied to the entire specimen $\left(200 \times 200 \mathrm{~mm}^{2}\right)$. The second option is to extract the displacements from the face of the specimen and apply them at the edge of a smaller (cropped) region of interest of $150 \times 200 \mathrm{~mm}^{2}$. Each approach presents advantages and drawbacks:

- Using the displacements from the loading plates, the simulation can be performed over the entire sample, hence the $\mathrm{BC}$ are as far as possible from the propagation zone. However, the presence of a more compliant medium between the sample and the $\mathrm{BC}$ (plates and glue layer) could be responsible for a discrepancy between actual and imposed BC. Moreover, in the vicinity of the glue interface, damage localization may occur and influence the macroscopic behavior.

- Using the displacements obtained for the resized sample, the $B C$ are closer to the propagation zone, thus their influence is increased. Moreover, since the displacement amplitudes are smaller, the signal-to-noise ratio is slightly lower. However, the data are extracted directly from the sample surface, thus the stiffness of the glue layer and the transmission of the load are no longer factors of influence. The resized sample is chosen as close as possible to the real specimen size but a reduction in height is necessary to avoid the influence of the U-shaped grips. For each case, the $\mathrm{BC}$ are extracted from both the upper and the lower part of the sample.

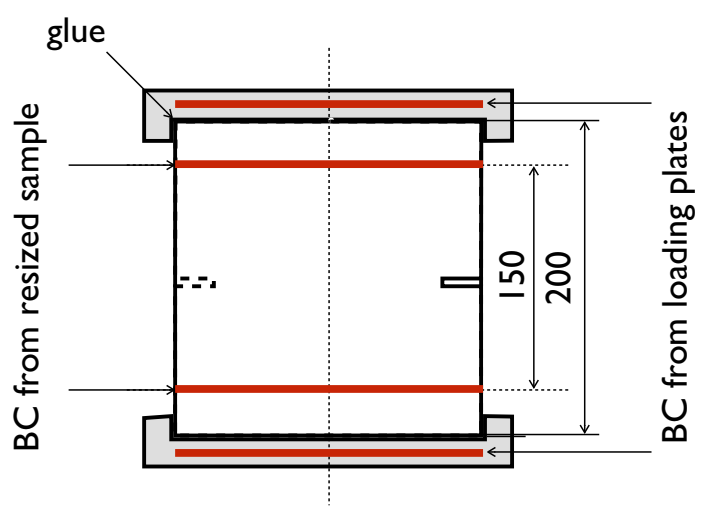

Fig. 8 Locations of the two types of BC 


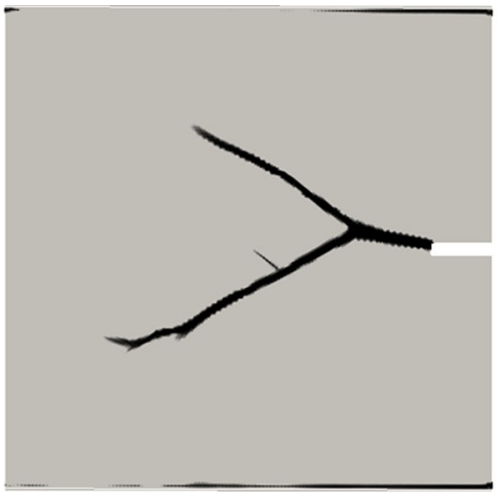

a

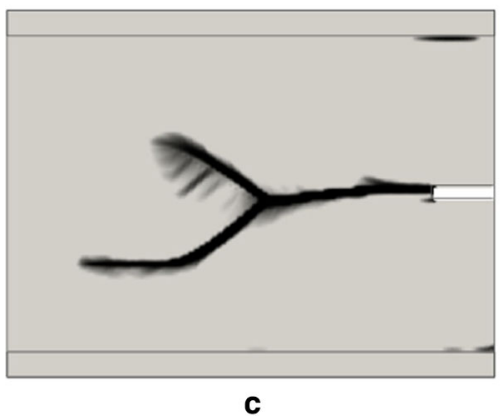

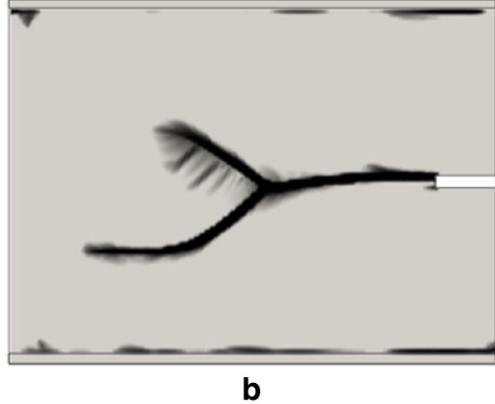

b

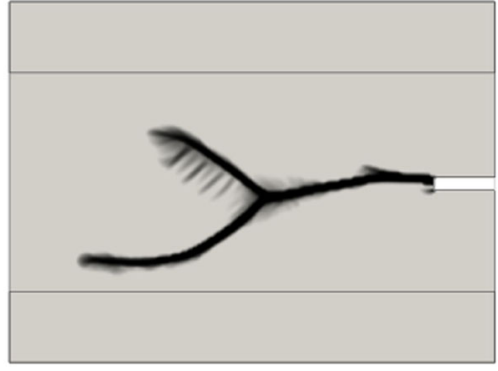

d

Fig. 9 High damage regions predicted by 2D simulations of test no 1 performed with the $B C$ extracted from the loading plates (a) and on resized samples with the BC extracted directly from the sample face (b). Results on resized sample with narrow (b), medium (c) and large (d) non-damageable bands are compared [25]

Using the $\mathrm{BC}$ measured on the loading plates and on the sample, 2D plane strain simulations are performed for the first test. The crack pattern obtained using the $\mathrm{BC}$ extracted from the loading plates (Fig. 9a) is not in good agreement with the experimental one, while the one obtained using the $\mathrm{BC}$ extracted from the sample (Fig. 9b) is much closer to reality.

\section{Size of the elastic layer}

Using the resized sample, a non-physical damage may appear close to the imposed Dirichlet condition. It is due to the noise or the fluctuations in the $\mathrm{BC}$ displacements which, however small, create large local stress concentrations and thus induce damage at the sample boundary. A non-damageable layer of same stiffness is implemented on both ends to prevent this problem. The influence of the height of these layers on the damage at the boundary and on the crack path is shown Fig. $9 \mathrm{~b}-\mathrm{d}$.

For narrow strips, damage is occurring at the interfaces (Fig. 9b), and for larger ones, the phenomenon is dampened (Fig. 9c) until no more damage initiates at the interface (Fig. 9d). The size of the bands has a very small influence on the crack pattern, but is important for a correct transmission of the load. The crack path obtained with the largest non-damageable bands (Fig. 9d) is in good agreement with the experimental data. One concludes that the experimental results can be reproduced by the models only if the $\mathrm{BC}$ extracted directly from the surface of the sample are used. 


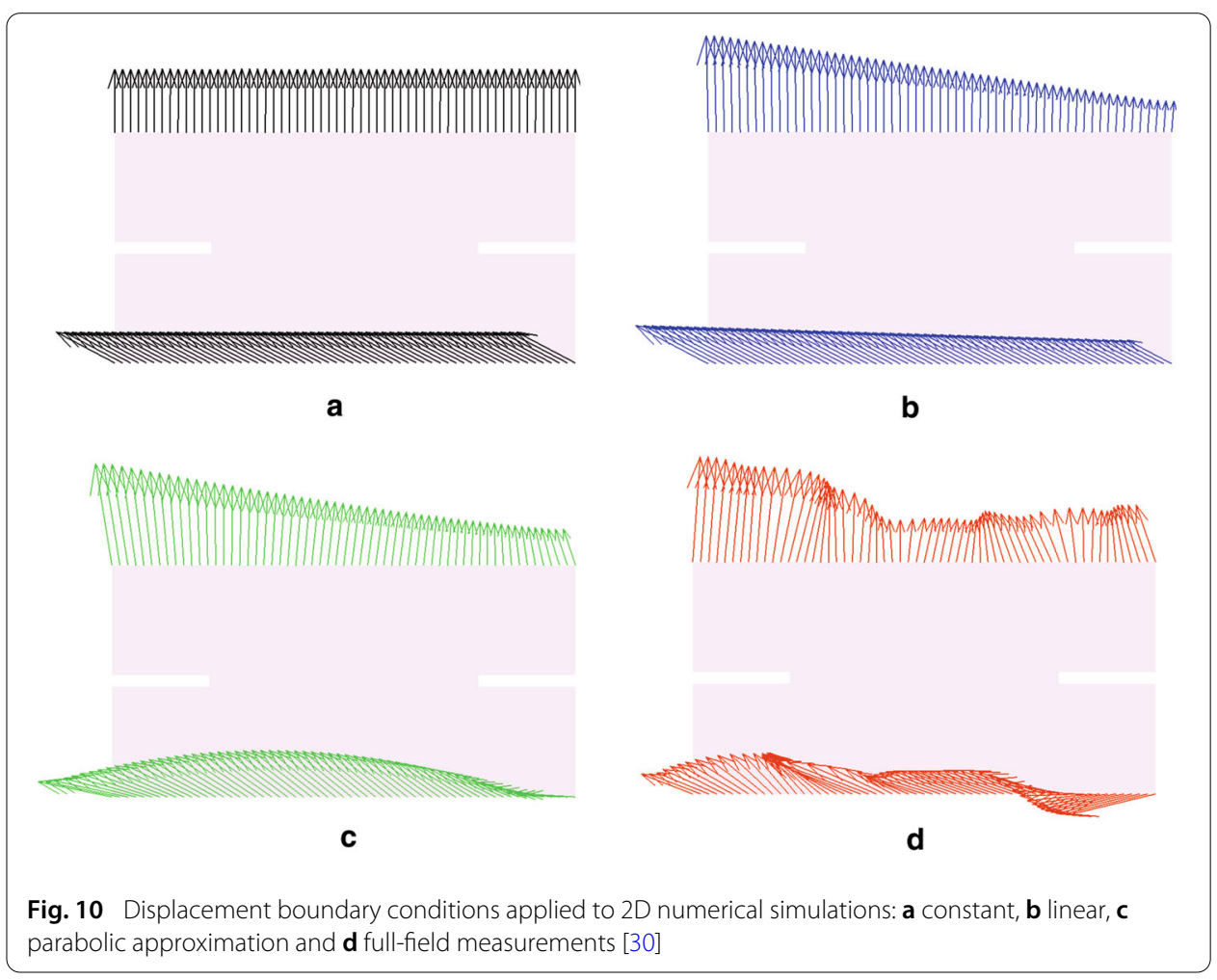

\section{Measured vs. simplified BC}

In [30] the sensitivity to the $\mathrm{BC}$ was studied on a different test (not one of the two proposed for the benchmark). In order to illustrate the importance of the $\mathrm{BC}$, the results are briefly presented in the following.

The test was performed on a double-notched sample by applying a proportional tensionshear displacement. Numerical simulations were performed using the measured BC, but also different approximations of the measured BC: constant, linear and parabolic ones obtained from least square regression (Fig. 10).

It was shown that for this proportional test, the sensitivity to the boundary conditions is crucial and the experimental results were accurately reproduced by numerical simulations only when accurate full-field BC were used. The results are very different both in terms of crack path (Fig. 11) and force-displacement graphs (Fig. 12). This high sensitivity is mostly due to the very small applied displacements $(\approx 20 \mu \mathrm{m})$ at complete fracture of the specimen.

The same analysis is conducted for the test no 1 of the benchmark, the $\mathrm{BC}$ being simplified by a linear approximation. One may observe that the crack path is not well reproduced when a linear approximation of the boundary conditions is used (Fig. 13). The most sensitive part is crack branching. Since several cracks are branching during the last loading step, only a very accurate $\mathrm{BC}$ modeling allows the right branching point to be recovered. Even though for the test no 1 the applied displacements are much larger than for the proportional tension-shear test $(>100 \mu \mathrm{m}$ ) only the full-field BC should still be used to perform numerical simulations. The main reason is the complexity of the crack pattern.

For the proportional tension-shear test and the benchmark test no 1 it was shown that the approximated boundary conditions do not correctly reproduce the experimental data. 

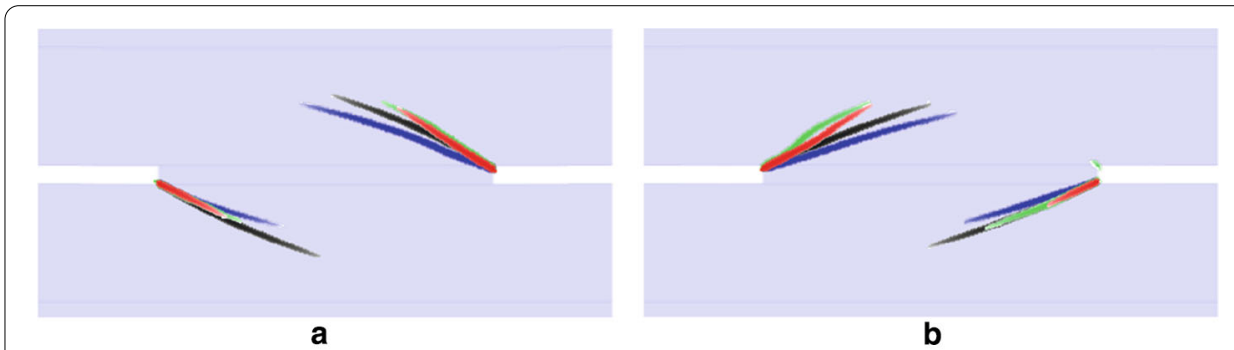

Fig. 11 Comparison between the crack paths predicted by the damage model, using constant (black), linear (blue), parabolic (green), BC approximations and the BC (red) measured during the test for each face of the sample [30]
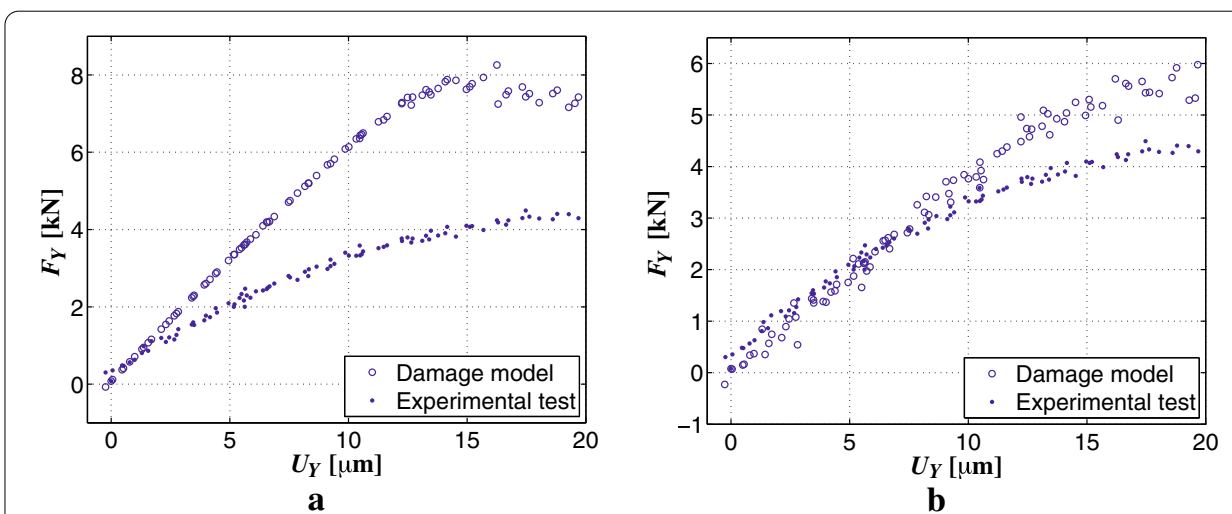

Fig. 12 Shear force for the proportional tension-shear test obtained with $2 \mathrm{D}$ simulation using averaged $\mathrm{BC}$ (a) and full-field BC (b) [30]

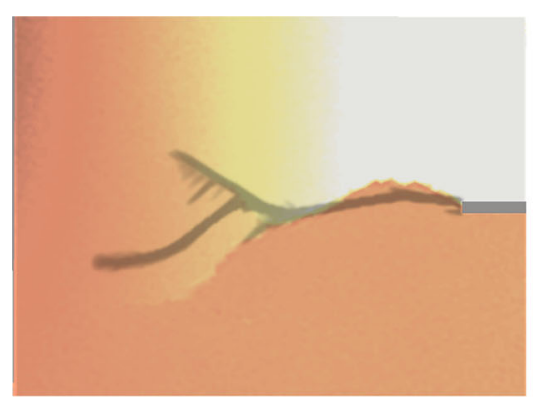

a

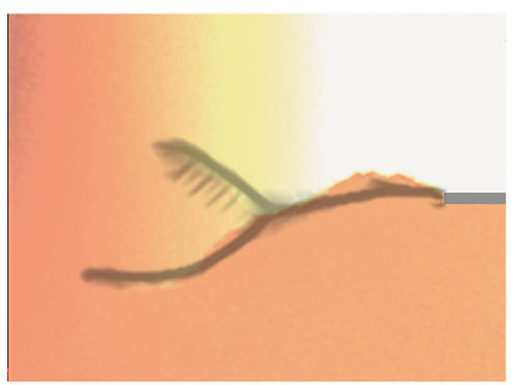

b

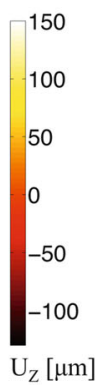

$\mathrm{U}_{\mathrm{Z}}[\mu \mathrm{m}]$

Fig. 13 Comparison for test no 1 between the crack paths predicted by the damage model (thick black lines) and the measured vertical displacement field: $\mathbf{a}$ model using a linear approximation of the $B C$ and $\mathbf{b}$ model using the measured $\mathrm{BC}$

For the benchmark test no 2, it can be simulated using a linear approximation of the $\mathrm{BC}$ as it leads to results very close to those obtained with the full-field data. In terms of crack patterns, only minor differences are observed (Fig. 14). The propagation angle of the right crack (i.e., $Y^{+}$) is slightly smaller than the one given by the full-field conditions. Moreover, for the same crack, propagation is shorter after the reorientation.

\section{Influence of the notch shape}

The shape of the notch is expected to have a strong influence on crack initiation for a quasi-brittle material. This question was addressed using the non-local damage model. 


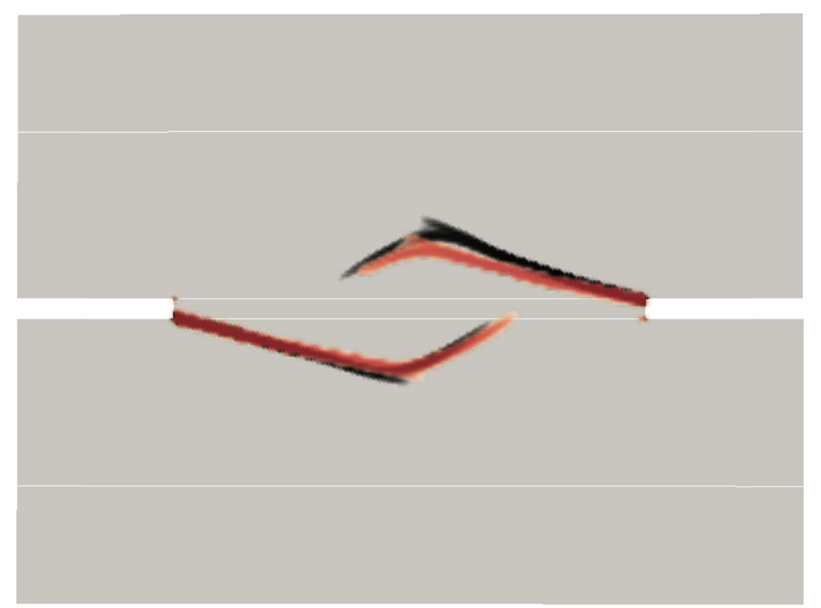

Fig. 14 Comparison for test no 2 between the crack paths predicted by the damage model for full-field BC (black thick lines) and for linear approximation BC (red thick lines)

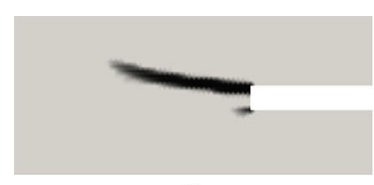

a

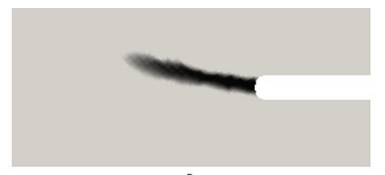

b

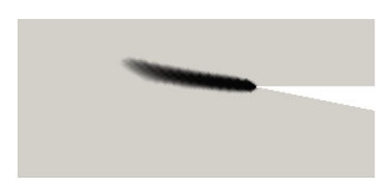

C

Fig. 15 High damage regions predicted by 2D simulations of test no 1 performed with different notch geometries: rectangular (a), rounded (b) and triangular (c) [25]

Simulations were performed with a rectangular notch shape (the shape used for all the numerical simulation presented in this work), a slightly rounded notch (consistent with the real shape of the notch, where the angles are not exactly $90^{\circ}$ because of the saw profile) and a triangular notch (Fig. 15), which is intentionally chosen to be far from the real one. The results are compared at the end of step 1 for test no 1 (before the first reorientation). Almost no difference is observed considering the propagation angle, and only the crack obtained with the rectangular notch is slightly longer that the other two. In conclusion, the shape of the notch has a minor influence on crack propagation.

\section{Conclusions}

This paper is a brief description of the CARPIUC benchmark proposed at the CFRAC2017 conference. It summarizes the experiment protocol and main results. Some preliminary attempts to model the tests using a non-local damage model were used to check the sensitivity of the simulation to different details in order to advise people who would be interested to take part to the benchmark. Questions regarding the use of kinematic boundary conditions, their fluctuations at small scale, or the accurate notch geometry for crack initiation were addressed and discussed. These results show that the use of accurate displacement data at the boundary is essential, whereas other aspects such as the notch geometry appear to be much less sensitive. This robustness makes the experimental results suited for a benchmark.

Research teams present at CFRAC2017 have confirmed the usability of the provided data, both for 2D and 3D computations, as well as for different approaches (damage 
models, phase-field description, discrete models,...). Each participating team will publish their results separately with all details. However, from a first and very preliminary round of comparison, some general conclusions may be listed:

- The exact initiation location and angle are difficult to obtain, but fortunately, even if the simulation fails to predict them, this has no further influence on the crack path;

- The angles of orientations (especially for the first 4 steps of test no 1 , and the first crack of test no 2) tend to be underestimated;

- The "dilemma" between branching and reorientation in test no 1 is a very difficult issue;

- The computed stiffness once the crack has already propagated and has changed direction is sometimes different from the measured one;

- The coalescence/linkup of test no 2 is very hard to simulate.

Thus, the initial goal of having experimental tests with a very dense set of measurements, including full-field displacement data, providing a well-posed problem for simulations has been met. Those two cases have been shown to be quite robust against some details which may be missing from the database. However, at the same time, the loading path has been chosen to lead to complex crack patterns, and hence be very discriminating. All those points make the CARPIUC benchmark a difficult but gratifying challenge.

\section{Authors' contributions}

AC developed the experimental protocol, performed the tests and the related numerical simulations. MP designed the testing machine and took (an active) part in to the experimental protocol elaboration and the tests setups. SR proposed the original concept of such tests and helped through numerous discussions during the whole project. JR helped to format, publish and organize the benchmark for CFRAC2017 conference. All authors read and approved the final manuscript.

\section{Author details}

${ }^{1}$ EDF-Recherche et Développement, EDF Lab Paris-Saclay, O2BC.20, 7, Boulevard Gaspard Monge, 91120 Paris, Palaiseau, France, ${ }^{2}$ LMT (ENS Paris-Saclay/CNRS/Univ. Paris-Saclay), 61 Avenue du Président Wilson, 94235 Paris, Cachan, France,

${ }^{3} \mathrm{GeM}$, Centrale Nantes, CNRS, 1 Rue de la Noë, 44321 Nantes, France.

\section{Acknowledgements}

The authors thanks the research teams that have participated to the benchmark sessions of CFRAC2017. The organizers of the benchmark are also grateful for the CFRAC2017 committee enthusiastic acceptance and their technical help.

Competing interests

The authors declare that they have no competing interests.

Availability of data and materials

Details and data are available online at https://cfrac2017.sciencesconf.org/resource/page/id/21

Consent for publication

Not applicable.

Ethics approval and consent to participate

Not applicable.

Funding

Not applicable.

\section{Publisher's Note}

Springer Nature remains neutral with regard to jurisdictional claims in published maps and institutional affiliations. 


\section{References}

1. Winkler B. Traglastuntersuchungen von unbewehrtenund bewehrten Betonstrukturen, Ph.D. thesis. Innsbruck: University of Innsbruck; 2001.

2. Iosipescu N. New accurate procedure for single shear testing of metals. J Mater. 1967;2:537-66.

3. Brokenshire DR. Torsional fracture tests. Ph.D. thesis. Cardiff: Cardiff University; 1996.

4. Gálvez JC, Elices M, Guinea GV, Planas J. Mixed mode fracture of concrete under proportional and nonproportional loading. Int J Fract. 1998;94:267-84.

5. Nooru-Mohamed MB, Schlangen E, van Mier JGM. Experimental and numerical study on the behavior of concrete subjected to biaxial tension and shear. Adv Cem Based Mater. 1993;1(1):22-37.

6. Jr JE, Bolander S Saito. Fracture analyses using spring networks with random geometry. Eng Fract Mech. 1998:61(5-6):569-91.

7. Cusatis G, Bazant ZP, Cedolin L. Confinement-shear lattice CSL model for fracture propagation in concrete. Comput Methods Appl Mech Eng. 2006;195(52):7154-71.

8. Desmorat R, Gatuingt F, Ragueneau F. Nonlocal anisotropic damage model and related computational aspects for quasi-brittle materials. Eng Fract Mech. 2007;74(10):1539-60.

9. Di Luzio G. A symmetric over-nonlocal microplane model M4 for fracture in concrete. Int J Solids Struct. 2007:44:4418-41.

10. Dong Y, Wu S, Xu SS, Zhang Y, Fang S. Fracture of concrete structure using simplified meshless method. Cem Concr Res. 2009;39(10):966-72.

11. Fichant S, La Borderie C, Pijaudier-Cabot G. A comparative study of isotropic and anisotropic descriptions of damage in concrete structures. In: Voyiadjis GZ, Ju W, Chaboche JL, editors. Applied mechanics, vol. 46. New York: Elsevier; 1998. p. 259-74.

12. Gasser TC, Holzapfel GA. 3D crack propagation in unreinforced concrete: a two-step algorithm for tracking 3D crack paths. Comput Methods Appl Mech Eng. 2006;195(37-40):5198-219.

13. Jirasek M, Grassl P. Evaluation of directional mesh bias in concrete fracture simulations using continuum damage models. Eng Fract Mech. 2008:85(8):1921-43.

14. Kozicki J, Tejchman J. Modelling of fracture process in concrete using a novel lattice model. Granular Matter. 2008;10:377-88

15. Lens LN, Bittencourt E, d'Avila VMR. Constitutive models for cohesive zones in mixed-mode fracture of plain concrete. Eng Fract Mech. 2009;76(14):2281-97.

16. Prochtel P, Haussler-Combe U. On the dissipative zone in anisotropic damage models for concrete. Int J Solids Struct. 2008;45(16):4384-406.

17. Réthoré J, Roux S, Hild F. Mixed-mode crack propagation using a hybrid analytical and extended finite element method. Comptes Rendus Mecanique. 2010;338(3):121-6.

18. Unger JF, Eckardt S, Konke C. Modeling of cohesive crack growth in concrete structures with the extended finite element method. Comput Methods Appl Mech Eng. 2007;196(41-44):4087-100.

19. Le Flohic J, Parpoil V, Bouissou S, Poncelet M, Leclerc H. A 3D displacement control by digital image correlation for the multiaxial testing of materials with a Stewart platform. Exp Mech. 2014;54(5):817-28.

20. Grediac M, Hild F. Full-field measurements and identification in solid mechanics, vol. 496. New York: Wiley; 2012.

21. Méthodes d'essais des ciments-Partie 1: détermination des résistances mécaniques (NF EN 196-1), AFNOR; 2006

22. Rilem TCS. Determination of the fracture energy of mortar and concrete by means of three-point bend tests on notched beams. Mater Struct. 1985;18(106):285-90.

23. Carpiuc-Prisacari A, Poncelet M, Kazymyrenko K, Leclerc H, Hild F. A complex full-field measured Nooru-Mohamed test performed with a 6 axis testing machine. Eng Fract Mech. 2017;176:1-22.

24. Nierenberger M, Poncelet M, Pattofatto S, Hamouche A, Raka B, Virely J-M. Multiaxial testing of materials using a Stewart platform: case study of the Nooru-Mohamed test. Exp Tech. 2012;38(2):74-83.

25. Carpiuc A. Innovative tests for characterizingmixed-mode fracture of concrete: from pre-defined to interactive and hybrid tests. Ph.D. thesis. Paris: University of Paris-Saclay; 2015.

26. Tomičević Z, Hild F, Roux S. Mechanics-aided digital image correlation. J Strain Anal Eng Design. 2013;48:330-43.

27. Nooru-Mohamed MB. Mixed-mode fracture of concrete: an experimental approach. Ph.D. thesis. Delft: Technische Universteit Delft; 1992.

28. Carpiuc-Prisacari A, Jailin C, Poncelet M, Kazymyrenko K, Leclerc H, Hild F. Experimental data base of mixed-mode crack propagation tests performed on concrete specimens with a 6 axes testing machine and full-field measurements. Part I : predefined loading tests. (in preparation)

29. Carpiuc-Prisacari A, Jailin C, Poncelet M, Kazymyrenko K, Leclerc H, Hild F. Experimental data base of mixed-mode crack propagation tests performed on concrete specimens with a 6 axes testing machine and full-field measurements. Part II : interactive loading tests. (in preparation)

30. Carpiuc-Prisacari A, Poncelet M, Kazymyrenko K, Hild F, Leclerc H. Comparison between experimental and numerical results of mixed-mode crack propagation in concrete: influence of boundary conditions choice. Cem Concr Res. 2017;100:329-40.

31. Lorentz E. A nonlocal damage model for plain concrete consistent with cohesive fracture. Int J Fract. 2017:207(2):123-59.

32. Lorentz E, Cuvilliez S, Kazymyrenko K. Convergence of a gradient damage model toward a cohesive zone model. Comptes Rendus Mécanique. 2011;339(1):20-6.

33. François M. A new yield criterion for the concrete materials. Comptes Rendus Mécanique. 2008;336(5):417-21. 